\title{
BMJ Global Health HIV self-testing: lessons learnt and priorities for adaptation in a shifting landscape
}

\author{
Ankur Gupta-Wright (D) ,,2 Ruanne V Barnabas, ${ }^{3}$ Heather Ingold (i) ,4 \\ Philippe Duneton, ${ }^{4}$ Ibrahim Abubakar ${ }^{1}$
}

To cite: Gupta-Wright A, Barnabas RV, Ingold $\mathrm{H}$, et al. HIV self-testing: lessons learnt and priorities for adaptation in a shifting landscape. BMJ Global Health 2021;6:e04418. doi:10.1136/ bmjgh-2020-004418

Handling editor Seye Abimbola

Received 9 November 2020 Revised 2 February 2021 Accepted 4 February 2021
Check for updates

(C) World Health Organization 2021. Licensee BMJ.

${ }^{1}$ Institute for Global Health, University College London, London, UK

${ }^{2}$ Clinical Research Department, London School of Hygiene \& Tropical Medicine, London, UK ${ }^{3}$ Department of Global Health, University of Washington, Seattle, Washington, USA ${ }^{4}$ Unitaid, Geneva, Switzerland

Correspondence to Ankur Gupta-Wright; a.gupta-wright@ucl.ac.uk

\section{INTRODUCTION}

HIV self-testing (HIVST) has shifted the paradigm for HIV testing, the first step in the care continuum. Although HIVST, whereby the person performs the test and interprets the results, was conceived early in the HIV epidemic, wider access to HIVST in low-income and middle-income countries (LMICs) is a recent phenomenon. A decade ago, large-scale HIVST use was limited due to concerns about accuracy, feasibility and safety. Through coordination between the WHO and implementation projects, including the Unitaid-funded HIV Self-Testing Africa (STAR) Initiative, HIVST is now part of the approach to HIV diagnosis in a growing number of high burden settings, with lessons for HIV programmes, other disease control efforts and the wider universal health coverage agenda. ${ }^{1}$

This shift to HIVST was driven by a need for better HIV diagnostic strategies, particularly in sub-Saharan Africa where under $50 \%$ of people living with HIV (PLHIV) knew their status in $2013 .{ }^{2}$ Despite no precedent for selftesting, no registered commercial assays and several implementation barriers, researchers and funders, set out to inform guidelines, policy development and regulatory frameworks. This effort shed light on the feasibility and effectiveness of large-scale implementation of HIVST by establishing preferred distribution channels, thereby generating demand. We describe key lessons learnt from HIVST in LMICs to tackle gaps in the diagnostic aspect of the HIV care cascade. We also discuss the future of HIVST in HIV, as UNAIDS 95-95-95 targets and ending AIDS approach, and the implications for other diseases, including COVID-19 and health systems more broadly.

\section{Summary box}

$\Rightarrow$ HIV self-testing has rapidly become part of the approach to HIV diagnosis in many high-burden settings through large-scale research and implementation projects, which have developed a robust evidence-base and tackled market and policy implementation barriers.

$\Rightarrow$ The changing HIV epidemic, with declining prevalence of undiagnosed HIV and increasing importance of key populations, will bring new challenges to HIV testing, which will need to reach these key populations.

$\Rightarrow$ Lessons learnt from implementation and scale-up of HIV self-testing are highly relevant to COVID-19, the looming epidemic of chronic disease and health system challenges more broadly.

\section{WHAT ARE THE KEY LESSONS LEARNT FROM HIVST?}

HIVST's success has been outlined by multicountry and multidisciplinary evidence demonstrating acceptability and accuracy across several delivery models and populations, with a focus on populations neglected by healthcare worker-based testing (especially men and younger people). ${ }^{34}$ Safety, a major concern from the outset, was demonstrated through extensive social science research, confirming minimal social and physical harms. ${ }^{5-7}$ Outcomes of HIVST research have also focused on 'real-world' implementation and relevance to policymakers, with randomised trials assessing linkage to HIV treatment, HIV prevention services for those who test HIV-negative and cost-effectiveness. ${ }^{8}$ Research on how populations want HIVST delivered, coupled with lessons learnt from implementation and evaluation, has informed scale-up in many high burden settings. ${ }^{9}$

Engagement of key stakeholders, such as manufacturers and regulatory bodies, has been vital to the rapid translation into 
guidelines, national policy and enabling a viable market. ${ }^{7}$ Examples include establishing a WHO prequalification system for HIVST (four HIVST kits have been prequalified) and market interventions, leading to price reductions and stimulating competition. Introducing and evaluating alternative products such as blood-based HIVST also widened the market, increased competition and led to further price reductions. However, the current costs of delivering HIVST remain relatively high and remains one of the major concerns for scaling-up outside donor-funded programmes. HIVST, building on scale-up of antiretroviral therapy and HIV viral load testing, ${ }^{10}$ could then provide a roadmap for other diagnostics, illustrating the evidence-base and market interventions needed to support rapid scale-up for other promising diagnostics that remain poorly implemented, for example, urine-based TB testing in PLHIV. ${ }^{12}$

\section{WHAT IS THE FUTURE OF HIVST IN A CHANGING EPIDEMIC?}

Through concerted global efforts, including the scale-up of HIVST, there has been substantial progress in HIV testing, prevention and care, resulting in approaching $90 \%$ of PLHIV knowing their status, being linked to care and taking treatment in many countries. ${ }^{13}$ Furthermore, prevalence of untreated HIV and HIV incidence is falling, which will have an important impact on the delivery of HIV testing services. ${ }^{14}$ Key populations (eg, sex workers and men who have sex with men) and their partners now account for over half of new HIV infections. While HIVST improves access to HIV diagnosis and care, how it can contribute to reaching remaining undiagnosed PLHIV in a changing epidemic is less clear.

Some considerations include implementing HIVST to focus on priority populations with the greatest gaps in testing. The strong evidence-base from HIVST can help select appropriate delivery and support mechanisms. However, these priority populations are likely to change as LMICs progress towards UNAIDS 95-95-95 targets and 'elimination', highlighting the need for a variety of tools, options and systems in place to adjust strategies quickly. For HIVST to translate to engagement with HIV services, beyond distribution of test kits, also requires systems to provide support with testing processes, such as phonebased support and virtual or local supervision.

There are also funding and cost-effectiveness considerations for HIVST as incidence declines. Delivering any screening test at scale is expensive. For HIVST, cost-effectiveness has been driven by the health benefits of early HIV diagnosis, which depends on high prevalence of undiagnosed HIV. ${ }^{15}$ However, evidence of cost-effectiveness of HIVST strategies focused on key population exist, even in low incidence settings. ${ }^{16} \mathrm{HIV}$ testing strategies that impact on HIV prevention more broadly, for instance by increasing uptake of pre-exposure prophylaxis, will also become an increasingly important consideration. Much HIVST remains donor-funded, but more sustainable and cheaper delivery models, including community-led or public-private partnerships, could facilitate transition to domestic health budgets, which will be necessary as donor-funding runs out. The different challenges of elimination need to be recognised with more generous thresholds and policies for funding, ${ }^{17}$ and awareness that conventional HIV testing services makes little sense unless accompanied by innovative solutions such as HIVST that better serve the needs of key populations. In addition to cost and funding, there remain some technology and evidence-gaps, particularly outside southern Africa and relating to linkage to care, that contribute to suboptimal implementation outside donor-funded programmes. ${ }^{18}$

\section{WHAT ARE THE IMPLICATIONS OF SELF-TESTING FOR COVID-19 AND OTHER DISEASES?}

The lessons learnt from HIVST have implications for other diseases. HIVST has developed alternative entry points for accessing healthcare in LMICs, for example community care providers and pharmacy or retail networks, who can distribute, supervise and support HIVST, and facilitate follow-up and linkage to services. COVID-19 has limited access to health facilities but testing for SARS-CoV-2 is crucial for outbreak control, surveillance, implementation of public health measures and isolating infectious cases. ${ }^{19}$ Point-of-care testing could help reduce pressures on already overburdened sample transport and laboratory infrastructure for PCR-based assays in LMICs. ${ }^{20}$

There is potential to replicate or harness HIV testing infrastructure for COVID-19 testing, tracing and supporting community management. HIVST already targets the most difficult to reach and underserved populations. The skillset of community HIV care providers could translate to new accurate and cheap COVID-19 point-of-care tests, including supporting both self-sampling and performing and interpreting tests (self-testing). ${ }^{21-23}$ Mechanisms to provide HIVST educational information could also be repurposed to provide public health messaging for COVID-19. However, the huge importance of funding, rapid evidence-base generation and collaboration at multilateral, industry, national and community levels should not be underestimated, and the role of self-testing in COVID-19 remains controversial. Funders and other agencies have already instigated volume guarantee agreements with manufacturers to ensure COVID-19 rapid diagnostic tests are aviable in LMICs. ${ }^{24}{ }^{25}$ Beyond HIV and COVID-19, there is also potential for repurposing these models for chronic disease self-management.

\section{WHAT ARE THE IMPLICATIONS OF SELF-TESTING FOR HEALTH} SYSTEMS?

Shifts from provider-driven care to individuals with chronic conditions driving their own care, such as self-monitoring for diabetes and hypertension, has 
been lacking from LMICs. ${ }^{26}$ Given that provision of facility-based healthcare at scale for a growing burden of chronic diseases is challenging within the current healthcare budgets of most LMICs, there is a need for another paradigm shift. HIVST is an important opportunity to push the self-management agenda in LMICs. ${ }^{27}$ Through building on efforts to shift diagnostics away from healthcare facilities, sustainable systems for community-based delivery and expanding access to person-centred treatment and prevention services can be developed more broadly. HIVST has required empowered and engaged communities, generated a demand for self-care and has led to shifts in perceptions of self-testing among healthcare workers; it will be vital to maintain this momentum to see benefits beyond the HIV epidemic. ${ }^{28}$

HIVST may also contribute to health system strengthening. Whereas the traditional mantra has been that vertical, disease focused interventions impair health system strengthening, the comprehensive approach to HIV care by donors has always aimed for HIV funding and services to benefit health systems and contribute to building universal health coverage. ${ }^{29}$ HIVST can support this, for example, through peer, partner and community-based delivery models and engagement of non-governmental and civil society organisations. COVID-19 has seen additional burdens and limitations placed on health systems globally. HIVST provides opportunities to both maintain HIV testing in this context, and opportunities beyond HIV to reduce the burden on health systems.

\section{CONCLUSION}

HIVST research and implementation initiatives, including STAR, through coordination between industry, policymakers, regulatory bodies, academia and implementing partners have developed a robust evidence-base, generated a viable market and led to rapid scale-up in several high-burden countries. Public health interventions have led to a shifting HIV landscape, and focused HIVST interventions will now be needed to reach key populations with poor engagement with services, or on generating demand for HIV prevention in order to be affordable and efficacious. More importantly, HIVST has accelerated healthcare simplification and transformation for communities and healthcare workers in these high HIV burden settings, providing an opportunity to transform infrastructure, delivery models, community engagement and demand for COVID-19 and the looming epidemic of chronic disease. However, more needs to be done to integrate and maintain self-testing within the standard-of-care, and secure government funding for HIVST and other conditions beyond.

Twitter Ankur Gupta-Wright @ankurgw

Contributors All authors contributed to the concept and development of the commentary. AG-W and IA wrote the first draft, and all authors reviewed the commentary and made substantial contributions to revisions. All authors reviewed and approved the final version.

Funding The authors have not declared a specific grant for this research from any funding agency in the public, commercial or not-for-profit sectors.

Competing interests $\mathrm{HI}$ and PD are employees of Unitaid, who funded the HIV Self-Testing Africa (STAR) Initiative.

Patient consent for publication Not required.

Provenance and peer review Not commissioned; externally peer reviewed.

Data availability statement There are no data in this work.

Open access This is an open access article distributed under the terms of the Creative Commons Attribution IGO License (CC BY NC 3.0 IGO), which permits use, distribution, and reproduction in any medium, provided the original work is properly cited. In any reproduction of this article there should not be any suggestion that WHO or this article endorse any specific organization or products. The use of the WHO logo is not permitted. This notice should be preserved along with the article's original URL.

Disclaimer: The author is a staff member of the World Health Organization. The author alone is responsible for the views expressed in this publication and they do not necessarily represent the views, decisions or policies of the World Health Organization.

\section{ORCID iDs}

Ankur Gupta-Wright http://orcid.org/0000-0002-5150-2970

Heather Ingold http://orcid.org/0000-0002-8714-8889

\section{REFERENCES}

1 World Health Organization. WHO recommends HIV Self-TestingEvidence update and considerations for success policy brief, 2019. Available: https://www.who.int/publications-detail/whorecommends-hiv-self-testing-evidence-update

2 United Nations Programme on HIV/AIDS (UNAIDS). The gap report, 2014. Available: https://www.aidsdatahub.org/sites/default/files/ publication/UNAIDS_Gap_report_en.pdf

3 Johnson CC, Kennedy C, Fonner V, et al. Examining the effects of HIV self-testing compared to standard HIV testing services: a systematic review and meta-analysis. J Int AIDS Soc 2017;20:21594.

4 Choko AT, MacPherson P, Webb EL, et al. Uptake, accuracy, safety, and linkage into care over two years of promoting annual SelfTesting for HIV in Blantyre, Malawi: a community-based prospective study. PLoS Med 2015;12:e1001873.

5 Offorjebe OA, Hoffman RM, Shaba F, et al. Acceptability of index partner HIV self-testing among HIV-positive clients in Malawi: A mixed methods analysis. PLoS One 2020;15:e0235008.

6 Bwalya C, Simwinga M, Hensen B, et al. Social response to the delivery of HIV self-testing in households: experiences from four Zambian HPTN 071 (PopART) urban communities. AIDS Res Ther 2020;17:32.

7 Ingold H, Mwerinde O, Ross AL, et al. The Self-Testing Africa (StAR) initiative: accelerating global access and scale-up of HIV self-testing. $J$ Int AIDS Soc 2019;22 Suppl 1:e25249-100.

8 Kelvin EA, Akasreku B. The evidence for HIV Self-Testing to increase HIV testing rates and the implementation challenges that remain. Curr HIVIAIDS Rep 2020;17:281-9.

9 Indravudh PP, Choko AT, Corbett EL. Scaling up HIV self-testing in sub-Saharan Africa. Curr Opin Infect Dis 2018;31:14-24.

10 Waning B, Kyle M, Diedrichsen E, et al. Intervening in global markets to improve access to HIV/AIDS treatment: an analysis of international policies and the dynamics of global antiretroviral medicines markets. Global Health 2010;6:9.

11 Peter T, Zeh C, Katz Z, et al. Scaling up HIV viral load - lessons from the large-scale implementation of HIV early infant diagnosis and CD4 testing. J Int AIDS Soc 2017;20 Suppl 7:e25008-15.

12 Singhroy DN, MacLean E, Kohli M, et al. Adoption and uptake of the lateral flow urine Lam test in countries with high tuberculosis and HIV/AIDS burden: current landscape and barriers. Gates Open Res 2020;4:24.

13 United Nations Programme on HIV/AIDS (UNAIDS). Global AIDS update 2020. Tackling entrenched inequalities to end epidemics; 2020.

14 World Health Organization. Consolidated guidelines on HIV testing services 2015, 2015. Available: https://apps.who.int/iris/bitstream/ handle/10665/179870/9789241508926_eng.pdf?sequence=1 LB Oojl 
15 Cambiano V, Johnson CC, Hatzold K, et al. The impact and costeffectiveness of community-based HIV self-testing in sub-Saharan Africa: a health economic and modelling analysis. J Int AIDS Soc 2019;22 Suppl 1:e25243.

16 Seguin M, Dodds C, Mugweni E, et al. Self-Sampling kits to increase HIV testing among black Africans in the UK: the HAUS mixedmethods study. Health Technol Assess 2018;22:1-158.

17 Sicuri E, Evans DB, Tediosi F. Can economic analysis contribute to disease elimination and eradication? A systematic review. PLoS One 2015;10:e0130603.

18 Choko AT, Jamil MS, MacPherson P, et al. Measuring linkage to HIV treatment services following HIV self-testing in low-income settings. $J$ Int AIDS Soc 2020;23:e25548.

19 Reddy KP, Shebl FM, Foote JHA, et al. Cost-Effectiveness of public health strategies for COVID-19 epidemic control in South Africa: a microsimulation modelling study. Lancet Glob Health 2021;9:e120-e129.

20 Ondoa P, Kebede Y, Loembe MM, et al. COVID-19 testing in Africa: lessons learnt. Lancet Microbe 2020;1:e103-4.

21 Atchison C, Pristerà P, Cooper E, et al. Usability and acceptability of home-based self-testing for SARS-CoV-2 antibodies for population surveillance. Clin Infect Dis 2020:ciaa1178.

22 Valentine-Graves M, Hall E, Guest JL, et al. At-home self-collection of saliva, oropharyngeal swabs and dried blood spots for SARSCoV-2 diagnosis and serology: Post-collection acceptability of specimen collection process and patient confidence in specimens. PLoS One 2020;15:e0236775.

23 McCulloch DJ, Kim AE, Wilcox NC, et al. Comparison of unsupervised home self-collected Midnasal swabs with clinician- collected nasopharyngeal swabs for detection of SARS-CoV-2 infection. JAMA Netw Open 2020;3:e2016382.

24 Clinton Health Access Initiative. Global partnership to make available $120 \mathrm{M}$ affordable, quality COVID-19 rapid tests for low- and middleincome countries. Available: https://www.clintonhealthaccess.org/ global-partnership-to-make-available-120m-affordable-qualitycovid-19-rapid-tests-for-low-and-middle-income-countries/ [Accessed 1 Dec 2020].

25 Unitaid. Cost of rapid COVID-19 tests halved as global investment ensures availability of high volumes for low- and middle-income countries, 2021. Available: https://unitaid.org/news-blog/costof-rapid-covid-19-tests-halved-as-global-investment-ensuresavailability-of-high-volumes-for-low-and-middle-income-countries/\# en [Accessed 1 Feb 2021]

26 Stephani V, Opoku D, Beran D. Self-Management of diabetes in sub-Saharan Africa: a systematic review. BMC Public Health 2018;18:s12889-018-6050-0:1-11.

27 World Health Organization (WHO). Framework on integrated, peoplecentred health services: report by the Secretariat, 2016. Available: http://apps.who.int/iris/bitstream/10665/174536/1/9789241564977 eng.pdf?ua=1,\%0Ahttp://apps.who.int/gb/ebwha/pdf_files/WHA69/ A69_39-en.pdf?ua=1\&ua=1\%0Ahttp://apps.who.int/gb/ebwha/pdf_ files/WHA69/A69 39-en.pdf?ua=1

28 Dzinamarira T, Kamanzi C, Mashamba-Thompson TP. Key stakeholders' perspectives on implementation and scale up of HIV self-testing in Rwanda. Diagnostics 2020;10:diagnostics10040194:194.

29 Jay J, Buse K, Hart M, et al. Building from the HIV response toward universal health coverage. PLoS Med 2016;13:e1002083. 